\title{
On the inclusion of heart failure as an outcome in hypertension studies
}

Thomas Kahan

Karolinska Institutet, Department of Clinical Sciences, Danderyd Hospital, Division of Cardiovascular Medicine, \& Department of Cardiology, Danderyd University Hospital Corporation, Stockholm, Sweden

You may be aware of this section to Hypertension News, which was introduced earlier this year. "To learn the ropes" means to find out how to do something, learning how a particular task or job is done, to acquire an expertise. The expression appeared in standard English some 200 years ago, and is most probably is of nautical origin, where basic skills handling the ropes on sailing ships were essential. We previously highlighted the possibilities and limitations of meta-

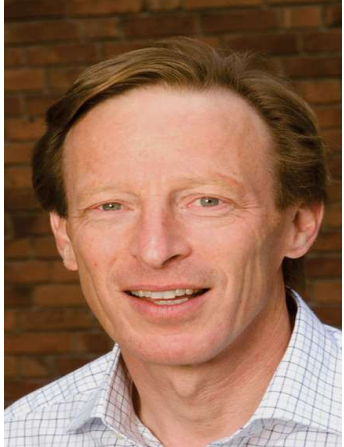
analyses for the assessment of studies in cardiovascular medicine. Another critical issue is to decide on the proper outcome to choose for large studies. A conservative and robust outcome is all-cause mortality. It is well defined, easy to understand for both health care providers and lay people, and can be applied across different diseases and other risks. However, as all-cause mortality (by definition) eventually will be $100 \%$ the time of follow up is important in the evaluation of results from clinical studies ${ }^{1}$.

Many hypertension and other cardiovascular trials also include acute non-fatal myocardial infarctions and nonfatal stroke into a composite endpoint. Both myocardial infarction and stroke are well defined, at least if subject to hospitalization. However, other cardiovascular events such as a transitory ischemic attack, angina pectoris, vascular procedures, or heart failure are more difficult to ascertain, in particular if there are no reliable objective biomarkers and/or a subjective evaluation and judgment included.

Hypertension is the most important attributable risk for incident heart failure. The prognosis for incident heart failure remains poor, with a 5-year mortality of approximately $50 \%$ from the first recorded diagnosis of heart failure ${ }^{2}$. Furthermore, heart failure patients are frequently hospitalized, with subsequent low quality of life and very high costs $^{3}$. Of note, some patients with heart failure seem to be hospitalized very often, while others my have only few hospitalizations for their disease during the course of time, suggesting that the cumulative number of hospitalizations may be more important than the time to the first event in studies of cardiovascular outcomes ${ }^{4}$. More recently, similar observations have been made also for patients with coronary artery disease ${ }^{5}$. But recurrent events are not independent. They cluster in a subgroup with many more than the average number of hospitalizations during a given time period. These and other considerations invalidate standard statistical techniques.

Heart failure is a clinical syndrome characterized by typical symptoms that may be accompanied by signs caused by a structural and/or functional cardiac abnormality ${ }^{6}$. However, symptoms and signs may be difficult to evaluate in obese individuals, in the elderly, in people with lower limb venous circulation abnormalities, and in patients with respiratory tract disease. An electrocardiogram, echocardiography, and natriuretic peptides often add to the diagnostic accuracy, but the diagnosis of new onset heart often remains difficult as symptoms and signs are non-specific, and values of natriuretic peptides and echocardiography findings are sometimes inconclusive ${ }^{7}$. Thus, heart failure as an outcome in large cardiovascular outcome studies remains an issue for discussion. For arguments pro and con, please see the excellent contributions elsewhere in this issue.

\section{Key References}

1. Swedberg K, Kjekshus J, Snapinn S. Long-term survival in severe heart failure in patients treated with enalapril. Ten year follow-up of CONSENSUS I. Eur Heart J 1999;20:136-9.

2. Zarrinkoub R, Wettermark B, Wändell P, et al. The epidemiology of heart failure, based on patient data for 2.1 million inhabitants in Sweden. Eur J Heart Fail 2013;15:995-1002. doi: 10.1093/eurjhf/hft064.

3. Mejhert M, Lindgren P, Schill O, et al. Health care consumption and cost expenditure in chronic systolic heart failure during an 8-12 year prospective follow up. Eur J Intern Med 2013,24:260-5. doi: 10.1016/j.ejim.2012.11.015.

4. Anker SD, McMurray JJ. Time to move on from 'time-to-first': should all events be included in the analysis of clinical trials? Eur Heart J 2012;33:2764-5. doi: 10.1093/eurheartj/ehs277.

5. Vasudevan A, Choi JW, Feghali GA. First and recurrent events after percutaneous coronary intervention: implications for survival analyses. Scand Cardiovasc J. 2019. [Epub ahead of print] doi: 10.1080/14017431.2019.1645349.

6. Ponikowski P, Voors AA, Anker SD, et al. 2016 ESC Guidelines for the diagnosis and treatment of acute and chronic heart failure: The Task Force for the diagnosis and treatment of acute and chronic heart failure of the European Society of Cardiology (ESC). Eur Heart J 2016;37:2129-2200. doi: 10.1093/eurheartj/ehw128.

7. Mejhert M, Kahan T. A management programme for suspected heart failure in primary care in cooperation with specialists in cardiology. Eur J 